\section{Condiciones de vida, situación de la salud y disponibilidad de servicios de salud en la frontera de Brasil: un enfoque geográfico}

\author{
Living conditions, health status and health \\ services availability along the Brazilian border: \\ a geographical approach
}

${ }^{1}$ Escola Politécnica de Saúde Joaquim Venâncio, Fundação Oswaldo Cruz, Rio de Janeiro, Brasil.

Correspondencia P. C. Peiter Escola Politécnica de Saúde Joaquim Venâncio, Fundação Oswaldo Cruz. Av. Brasil 4365, Rio de Janeiro, $R J$ 21040-900, Brasil. ppeiter@fiocruz.br

\begin{abstract}
The aim of this study was to identify critical health care areas along the Brazilian border. The distribution of health resources was analyzed by municipality and border area in 1999-2000 and subsequently analyzed according to living conditions, health status, geographic accessibility, and other aspects of the border dynamics. Secondary data were used from large national health and geographic databases, complemented by primary data collected in the field. Geographic information systems were used to analyze the data and to produce maps. The border area was heterogeneous in terms of the abovementioned characteristics. The municipalities along the northern portion of the border strip showed the most unfavorable conditions and thus constitute the most critical area for health care. The central portion showed an intermediate situation, with a balanced proportion of subregions in critical versus non-critical situations. The southernmost portion showed a satisfactory situation in all its sub-regions. Health care heterogeneity along the border showed the need for a specific focus, taking the different sub-regions and border dynamics into account.
\end{abstract}

Border Areas; Health Services; Social Conditions; Health Care (Public Health)
Paulo Cesar Peiter 1

\section{Introducción}

La frontera internacional de Brasil fue considerada por el sector de la salud durante muchos años como un área de control de tránsito de personas y mercancías, realizado en puestos fronterizos de vigilancia sanitaria, localizados en puntos considerados estratégicos del límite internacional. No existía una política específica para esta extensa región que considerara las particularidades de las poblaciones que viven en estas áreas (población fronteriza), ni su diversidad. Este vacío sólo empieza a superarse de forma más articulada, a partir de la constitución del MERCOSUR, en 1991, que instituye el proceso de integración económica entre Brasil, Argentina, Paraguay y Uruguay. Este proceso suscitó la aparición de una serie de denominadores comunes apremiantes para todos los estados en cuestión, como la utilización de los servicios de salud locales por parte de ciudadanos de la frontera 1 . Tras un largo periodo de discusiones y negociaciones se creó, en el ámbito del Ministerio de la Salud de Brasil, el Programa Integrado de Salud de las Fronteras - SIS-Fronteras (Ministerio de la Salud, Decreto GM no. 1.120, de junio de 2005). Uno de sus objetivos es establecer un sistema de compensación financiera para los municipios situados a lo largo de la frontera internacional que reciben flujos regulares de pacientes de los países vecinos.

Con ello, se inaugura una nueva fase de las políticas de salud en las fronteras brasileñas, que 
exige un esfuerzo de investigación e indagaciones para la comprensión de esta peculiar región, sus necesidades y problemas. Es a este esfuerzo al que el presente artículo se adhiere.

\section{Frontera internacional y salud - elementos teórico-conceptuales}

La zona de frontera internacional es una región con características propias con efectos en el comportamiento de sus poblaciones 2,3 , y consecuentemente en el proceso salud-enfermamiento. El límite político contenido en ella divide conjuntos socio-espaciales diferentes. Esta separación es producto y/o causante de asimetrías que se concretan en diferencias en el desarrollo económico, legislación, cultura, oferta de servicios (incluyendo los de salud), entre otros 4 .

Las poblaciones de las zonas fronterizas viven estas asimetrías de modo particular, estableciendo estrategias para superar los obstáculos puestos por el límite internacional y disfrutar de las oportunidades ofrecidas ante la proximidad con el "extranjero".

La movilidad transfronteriza es una estrategia de las poblaciones, pero también es un problema para la vigilancia en el ámbito de la salud, ya que dificulta los registros de salud (casos de enfermedades, nacimientos y muertes); el seguimiento del tratamiento de pacientes; la planificación y la implementación de acciones en salud (prevención, control y monitorización de enfermedades transmisibles); y la magnitud de los servicios; además de hacer ineficaces acciones territoriales de salud como la inmunización, el control de vectores, y el control ambiental por regla general 5,6. Por estos motivos, la frontera debe ser vista como un área particularmente vulnerable para la salud. Sin embargo, no basta reconocer la vulnerabilidad de la frontera, se tiene que considerar también las especificidades de cada región para que se instituyan políticas públicas de salud más adecuadas.

Es esta la propuesta de este artículo: ampliar el conocimiento de la región fronteriza, su situación de salud, condiciones de vida y disponibilidad de recursos humanos e infraestructura de atención de salud, apuntando las especificidades de cada uno de sus segmentos.

\section{La Franja Fronteriza de Brasil y sus particularidades}

Para el análisis de la región fronteriza internacional de Brasil se utilizó la división geográfica de la Franja de Frontera, establecida en 150km de ancho, paralela a la línea divisoria terrestre del te- rritorio nacional (Ley $n^{\circ}$. 6.634, del 2 de mayo de 1979). La Franja de Frontera se sitúa en la zona de frontera, que es una región más amplia que abarca las Franja de Frontera a cada lado del límite internacional 7 .

La Franja de Frontera brasileña estaba constituida en el año 2000 por 569 municipios, situados total o parcialmente dentro de estos límites. Con $15.719 \mathrm{~km}$ de extensión ocupaba un área de $2.300 .000 \mathrm{~km}^{2}(27,6 \%$ del territorio nacional) y comprendía casi 10 millones de habitantes (6\% de la población nacional).

La gran diversidad existente a lo largo de la frontera permite la identificación de tres macrorregiones bien diferenciadas y denominadas arcos norte, central y sur, a su vez subdivididas en 19 sub-regiones (seis en el norte, ocho en el central y cinco en el sur-Figura 1).

$\mathrm{Su}$ diversidad proviene de diferencias en las condiciones físicas (clima, relieve, vegetación, hidrografía, etc.), pero también demográficas, sociales, económicas y culturales. Se observan incluso diferencias en la "permeabilidad" de la frontera, es decir, en los tipos e intensidad de las interacciones transfronterizas que se producen a lo largo de la misma. Este último aspecto resulta en un mayor o menor intercambio de pacientes entre los servicios de salud instalados en la zona fronteriza, además de condicionar las acciones de control de enfermedades realizadas a cada lado del límite internacional, con efectos indirectos en la vulnerabilidad de sus poblaciones. Por este motivo hay que analizar cada segmento de la frontera brasileña, identificar sus problemas y necesidades particulares y localizar los espacios críticos para la atención de salud.

\section{Metodología}

Con el fin de caracterizar la Franja de Frontera e identificar sus espacios críticos para la asistencia de salud se realizó una estratificación geográfica a través de indicadores de condiciones de vida, situación de salud, disponibilidad de recursos humanos e infraestructura de salud, accesibilidad geográfica y una evaluación de las interacciones transfronterizas.

La estratificación geográfica se ejecutó en dos niveles: macrorregional (arcos) y mesorregional (sub-regiones), a partir de la regionalización de la Franja de Frontera elaborada por el Grupo Retis/ Universidade Federal de Río de Janeiro para el Programa de Desarrollo de la Franja de Frontera del Ministerio de Integración Nacional 8 . Este recurso metodológico fue necesario, dada la gran extensión de la frontera brasileña, que atraviesa áreas con características ambientales y sociales muy 


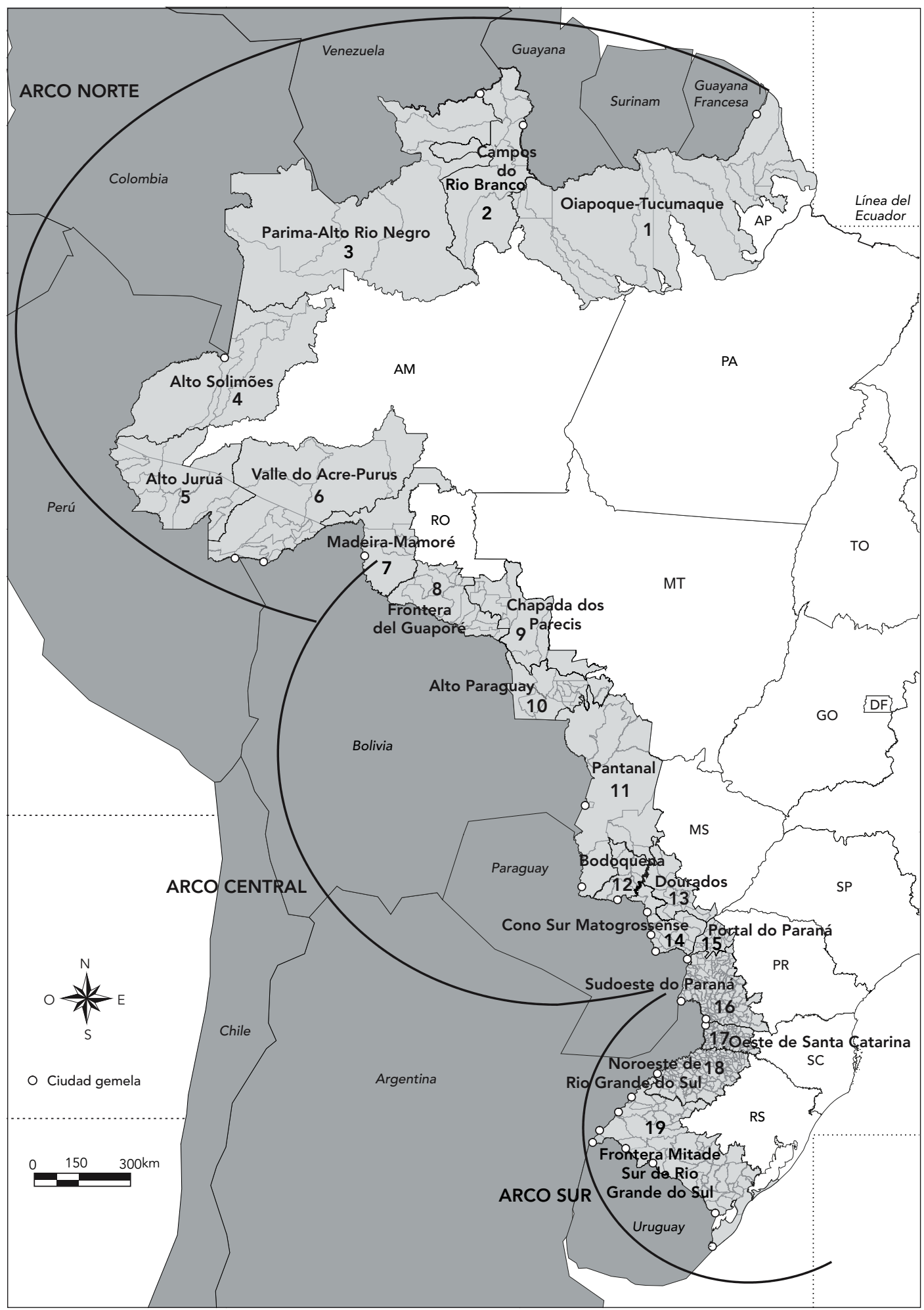

Fuente: Ministerio de Integración Regional; Grupo Retis/Universidad Federal de Río de Janeiro, 2005. 
distintas. Esta regionalización tuvo como objetivos delimitar áreas relativamente homogéneas en función de dos grandes vectores: el desarrollo económico regional y la identidad cultural, elementos que también condicionan el comportamiento de los flujos poblacionales en la región.

Para la caracterización de cada segmento de la frontera y de los servicios de salud disponibles en ellos se crearon indicadores de las condiciones materiales de vida, accesibilidad y disponibilidad de servicios de salud, completados con datos de poblamiento, migración e información recogida sobre el terreno en cinco puntos distintos de la frontera entre los años 2001 a 2006, a saber: en las ciudades gemelas de Tabatinga (Brasil) y Leticia (Colombia); Pacaraima (Brasil) y Santa Elena de Uairén (Venezuela); Bonfim (Brasil) y Lethem (Guayana); Brasiléia/Epitaciolândia (Brasil) y Cobija (Bolivia); Plácido de Castro (Brasil) y Montevideo (Bolivia); Cáceres (Brasil) y San Matías (Bolivia) y Corumbá (Brasil) y Puerto Suárez (Bolivia) y sus respectivas zonas fronterizas.

El índice de condiciones de vida intentó abarcar la dimensión económica, educacional y de vivienda de las poblaciones fronterizas y se compone de las siguientes variables: proporción de personas que viven en domicilios con renta familiar per cápita menor de 1/4 del salario mínimo); proporción de personas con más de 25 años con menos de 4 años de estudio; proporción de personas con 15 años o más analfabetas; proporción de personas que viven en domicilios sin agua corriente y lavabo; proporción de personas que viven en domicilios con más de 2 personas por habitación.

A partir de este índice, los municipios de la franja se estratificaron en tres clases de condiciones materiales de vida: desfavorable, intermedia y favorable, mediante el método del natural break. Estas variables se obtuvieron en las bases de datos del Instituto Brasileño de Geografía y Estatística (IBGE) 9 .

Para la caracterización de la situación de salud en la Franja de Frontera se utilizaron los indicadores de esperanza de vida al nacer (Instituto de Investigaciones Económicas Aplicadas. Datos macroeconómicos y regionales. http://www.ipeadata.gov.br, accedido el 10/Oct/2004) y mortalidad infantil (SIM/SINASC, 1999) obtenidos en el sitio web del Departamento de Informática del SUS (DATASUS. Informaciones de salud. http://www. datasus.gov.br, accedido el 10/Oct/2004).

Para la caracterización de la distribución geográfica de la capacidad de los servicios de salud instalados en la Franja de Frontera se utilizaron las siguientes variables: número de hospitales y porcentaje de camas por mil habitantes; número de ambulatorios y porcentaje de ambulatorios por 10 mil habitantes 10 .
La caracterización de la distribución geográfica de recursos humanos en salud en la Franja de Frontera fue realizada a partir de las siguientes variables: médicos por mil habitantes; enfermeros por mil habitantes y odontólogos por mil habitantes.

La caracterización de la cobertura de salud en los municipios de la Franja de Frontera se efectuó a partir de un índice de cobertura de salud creado con las siguientes variables: cobertura de vacunación (general - SIM-PNI, 2000); proporción de la población cubierta por los Programa Salud de la Familia - PSF y Programa de Agentes Comunitarios de Salud - PACS (SIAB, 2000), y media anual de consultas médicas en el periodo 1998-2002 (Sistema de Información Ambulatoria/ SUS, 2004). Este índice permitió estratificar las sub-regiones de la frontera en tres clases: cobertura alta, cobertura media y cobertura baja (todos estos bancos de datos fueron accedidos a través del DATASUS).

Otro aspecto que influencia la situación de salud en la frontera es la accesibilidad geográfica a los servicios disponibles, en particular el acceso de las poblaciones fronterizas rurales a los respectivos núcleos municipales, donde se concentran los recursos de salud, y de éstas hasta otros centros de referencia de la salud regionales, donde hay mayor disponibilidad de recursos para la atención de complejidad media y alta. La mitad de los municipios de la Franja de Frontera posee menos de 10 mil habitantes y muchos se sitúan en regiones remotas y desprovistas de transportes rápidos y de bajo coste, lo que convierte la accesibilidad geográfica a estos municipios en un elemento esencial para la atención de complejidad media y alta, ya que estos niveles de atención difícilmente se encuentran en municipios de pequeña población, por razones de viabilidad económica. Para medir el grado de accesibilidad de cada municipio de la región se creó un índice compuesto por las siguientes variables: número de autovías, ferrocarriles (Guia 4 Rodas) 11, ríos navegables (Agencia Nacional de Transportes Fluviales. http://www.antaq.gov.br, accedido en Oct/2004) y aeropuertos (INFRAERO. http:// www.infraero.gov.br, accedido en Oct/2004) existentes en el territorio municipal, además del coste medio del transporte desde el núcleo municipal hasta la capital estatal más cercana, donde en teoría se encuentra la atención de mayor nivel de complejidad.

El análisis conjunto de los indicadores arriba descritos con las características y dinámica de poblamiento, aspectos económicos y geográficos de cada sub-región permitieron una aproximación a la situación de la asistencia a la salud en la Franja Fronteriza. 


\section{Resultados}

El poblamiento es el primer aspecto que llama la atención dentro de la gran diversidad fronteriza. El arco norte es el mayor con $1.524 .156 \mathrm{~km}^{2}$ (66,7\% del área de la franja), por otro lado es el menos poblado de todos (menos de 1 habitante por $\mathrm{km}^{2}$ ). Su población de 1.284.938 habitantes representa sólo un 15,7\% del total de la franja, mientras que un $37,8 \%$ vive en áreas rurales 9, es decir, en aldeas, asentamientos rurales, pequeñas agrupaciones en las riberas de los ríos (ribereños) y en el interior del bosque (Tabla 1).

La población urbana vive en pequeños núcleos municipales, o en las dos capitales provinciales situadas en el arco norte (Boa Vista y Rio Branco). El porcentaje de poblaciones indígenas es significativo, y supera la población no-indígena en algunos municipios. Este grupo poblacional es considerado altamente vulnerable, pues su modo de vida está constantemente amenazado por los nuevos procesos de ocupación de la región.
Otros grupos con presencia destacada en la región y que se caracterizan por su elevada movilidad, son los madereros, comerciantes y buscadores de metales y piedras preciosas que transitan por la zona de frontera norte 12 .

La movilidad poblacional en la región proviene de la actividad extractora, que ocupa a la mayor parte de la población económicamente activa, de las actividades comerciales y de la implantación de asentamientos rurales nuevos que atraen inmigrantes de diversos Estados a la región. La subregión Oiapoque-Tumucumaque (SR-1) atrajo, en los últimos años de la década de los 90, buscadores de metales y piedras preciosas interesados en emigrar a países vecinos como las Guayanas y Surinam, donde existen mayores oportunidades para la actividad de minería de piedras y metales preciosos. A su vez, la sub-región Campos de Río Branco (SR-2) atrajo agricultores inmigrantes a los nuevos asentamientos rurales allí implantados. Estos inmigrantes son grupos de alta vulnerabilidad, representando un reto más para la atención de salud en la región.

Tabla 1

Franja de Frontera (Brasil): aspectos del poblamiento de las sub-regiones, 2000.

\begin{tabular}{|c|c|c|c|c|c|c|c|c|c|c|c|c|}
\hline \multirow[t]{2}{*}{ Sub-región } & \multicolumn{12}{|c|}{ Aspectos del poblamiento } \\
\hline & $\begin{array}{l}\text { Muni- } \\
\text { cipios }\end{array}$ & $\begin{array}{l}\text { Área } \\
\left(\mathrm{km}^{2}\right)\end{array}$ & $\begin{array}{l}\text { Población } \\
\text { (2000) }\end{array}$ & $\begin{array}{c}\text { Población } \\
\text { urbana }\end{array}$ & $\begin{array}{c}\text { Población } \\
\text { rural }\end{array}$ & $\begin{array}{l}\text { Densidad } \\
\text { rural }\end{array}$ & $\begin{array}{c}\text { Emigrantes } \\
\text { recientes }\end{array}$ & $\begin{array}{c}\text { Emigrantes } \\
\text { recientes } \\
\text { (\%) }\end{array}$ & $\begin{array}{l}\text { Población } \\
\text { indígena }\end{array}$ & $\begin{array}{c}\text { Población } \\
\text { indígena } \\
\text { (\%) }\end{array}$ & $\begin{array}{c}\text { Tierras } \\
\text { indígena }\end{array}$ & $\begin{array}{c}\text { Tierras } \\
\text { indígenas } \\
\text { (\%) }\end{array}$ \\
\hline Arco Norte & 71 & 1.524 .159 & 1.545 .938 & 952.915 & 583.831 & - & 48.347 & - & 119.890 & - & 495.663 & - \\
\hline $\begin{array}{l}\text { Oiapoque-Tumu- } \\
\text { cumaque (SR-1) }\end{array}$ & 18 & 409.431 & 298.676 & 176.379 & 121.978 & 0,30 & 9.416 & 8,29 & 9.430 & 3,16 & 117.113 & 28,60 \\
\hline $\begin{array}{l}\text { Campos do Rio } \\
\text { Branco (SR-2) }\end{array}$ & 9 & 138.049 & 280.057 & 228.222 & 51.835 & 0,40 & 26.602 & 18,27 & 23.475 & 8,38 & 36.613 & 26,52 \\
\hline $\begin{array}{l}\text { Parima-Alto } \\
\text { Rio Negro (SR-3) }\end{array}$ & 7 & 421.072 & 102.198 & 36.025 & 66.846 & 0,20 & 2.016 & 9,62 & 38.583 & 37,75 & 197.738 & 46,96 \\
\hline Alto Solimões (SR-4) & 8 & 202.052 & 171.752 & 77.268 & 89.769 & 0,40 & 1.372 & 6,30 & 33.496 & 19,50 & 104.811 & 51,87 \\
\hline Alto Juruá (SR-5) & 13 & 126.749 & 214.943 & 98.326 & 114.883 & 0,90 & 1.558 & 6,36 & 9.666 & 4,50 & 19.694 & 15,53 \\
\hline $\begin{array}{l}\text { Valle do Acre- } \\
\text { Purus (SR-6) }\end{array}$ & 16 & 226.806 & 478.312 & 336.695 & 138.520 & 0,60 & 7.383 & 5,02 & 5.240 & 1,10 & 19.694 & 8,68 \\
\hline $\begin{array}{l}\text { \% Franja de } \\
\text { Frontera }\end{array}$ & 12,5 & 66,73 & 15,69 & 13,71 & 20,19 & - & 24,66 & - & 57,35 & - & 89,26 & - \\
\hline Arco Central & 95 & 513.919 & 2.122 .951 & 1.537 .109 & 583.069 & - & 75.443 & - & 58.334 & - & 59.661 & - \\
\hline $\begin{array}{l}\text { Madeira-Mamoré } \\
\text { (SR-7) }\end{array}$ & 5 & 76.280 & 424.152 & 331.900 & 92.252 & 1,20 & 19.784 & 9,43 & 4.505 & 1,06 & 16.004 & 20,98 \\
\hline $\begin{array}{l}\text { Frontera del } \\
\text { Guaporé (SR-8) }\end{array}$ & 14 & 58.491 & 231.128 & 108.600 & 122.528 & 2,10 & 10.894 & 6,54 & 1.302 & 0,56 & 14.336 & 24,51 \\
\hline $\begin{array}{l}\text { Chapada dos } \\
\text { Parecis (SR-9) }\end{array}$ & 12 & 74.349 & 219.626 & 165.792 & 54.196 & 0,73 & 12.286 & 8,11 & 3.342 & 1,52 & 26.334 & 35,42 \\
\hline Alto Paraguai (SR-10) & 16 & 53.451 & 192.732 & 122.839 & 122.839 & 2,30 & 5.558 & 4,98 & 1.202 & 0,62 & 975 & 1,82 \\
\hline Pantanal (SR-11) & 10 & 167.335 & 348.349 & 267.329 & 25.367 & 0,20 & 6.486 & 6,63 & 14.963 & 4,30 & 929 & 0,60 \\
\hline
\end{tabular}

(continua) 


\begin{tabular}{|c|c|c|c|c|c|c|c|c|c|c|c|c|}
\hline \multirow[t]{2}{*}{ Sub-región } & \multicolumn{12}{|c|}{ Aspectos del poblamiento } \\
\hline & $\begin{array}{l}\text { Muni- } \\
\text { cipios }\end{array}$ & $\begin{array}{l}\text { Área } \\
\left(\mathrm{km}^{2}\right)\end{array}$ & $\begin{array}{l}\text { Población } \\
\text { (2000) }\end{array}$ & $\begin{array}{c}\text { Población } \\
\text { urbana }\end{array}$ & $\begin{array}{c}\text { Población } \\
\text { rural }\end{array}$ & $\begin{array}{l}\text { Densidad } \\
\text { rural }\end{array}$ & $\begin{array}{c}\text { Emigrantes } \\
\text { recientes }\end{array}$ & $\begin{array}{l}\text { Emigrantes } \\
\text { recientes } \\
(\%)\end{array}$ & $\begin{array}{l}\text { Población } \\
\text { indígena }\end{array}$ & $\begin{array}{l}\text { Población } \\
\text { indígena } \\
\text { (\%) }\end{array}$ & $\begin{array}{c}\text { Tierras } \\
\text { indígena }\end{array}$ & $\begin{array}{c}\text { Tierras } \\
\text { indígenas } \\
\text { (\%) }\end{array}$ \\
\hline Bodoquena (SR-12) & 7 & 22.673 & 100.064 & 74.697 & 25.367 & 1,10 & 2.307 & 5,59 & 1.902 & 1,90 & 57 & 0,25 \\
\hline $\begin{array}{l}\text { Dourados (SR-13) } \\
\text { Cono Sur- }\end{array}$ & 16 & 32.275 & 354.747 & 279.889 & 74.238 & 2,30 & 9.401 & 5,54 & 14.229 & 4,01 & 89 & 0,28 \\
\hline $\begin{array}{l}\text { Matogrossense } \\
\text { (SR-14) }\end{array}$ & 15 & 29.065 & 252.153 & 186.063 & 66.282 & 2,30 & 8.727 & 7,45 & 16.889 & 6,70 & 937 & 3,20 \\
\hline $\begin{array}{l}\text { \% Franja de } \\
\text { Frontera }\end{array}$ & 16,7 & 22,50 & 21,54 & 22,12 & 20,16 & - & 38,48 & - & 27,90 & - & 10,74 & - \\
\hline Arco Sur & 403 & 245.930 & 6.186 .840 & 4.460 .347 & 1.724 .767 & - & 72.246 & - & 30.826 & - & 0,00 & - \\
\hline $\begin{array}{l}\text { Portal do } \\
\text { Paraná (SR-15) }\end{array}$ & 24 & 12.277 & 301.649 & 224.049 & 77.600 & 6,30 & 6.256 & 3,64 & 627 & 0,21 & - & - \\
\hline $\begin{array}{l}\text { Sudoeste del } \\
\text { Paraná (SR-16) }\end{array}$ & 115 & 52.871 & 1.956 .738 & 1.420 .628 & 536.718 & 10,20 & 37.100 & 3,62 & 8.522 & 0,44 & - & - \\
\hline $\begin{array}{l}\text { Oeste de Santa } \\
\text { Catarina (SR-17) } \\
\text { Noroeste de Rio }\end{array}$ & 82 & 16.010 & 764.139 & 455.380 & 308.759 & 19,30 & 14.785 & 4,32 & 5.447 & 0,71 & - & - \\
\hline $\begin{array}{l}\text { Grande do Sul } \\
\text { (SR-18) } \\
\text { Frontera Mitad }\end{array}$ & 133 & 45.363 & 1.384 .259 & 885.937 & 495.522 & 10,90 & 5.935 & 1,34 & 11.506 & 0,83 & - & - \\
\hline $\begin{array}{l}\text { Sur de Rio Grande } \\
\text { do Sul (SR-19) }\end{array}$ & 49 & 119.409 & 1.780 .055 & 1.474 .353 & 306.168 & 2,60 & 8.170 & 1,97 & 4.724 & 0,27 & - & - \\
\hline $\begin{array}{l}\text { \% Franja de } \\
\text { Frontera }\end{array}$ & 70,8 & 10,77 & 62,77 & 64,17 & 59,65 & - & 36,85 & - & 14,75 & - & 0,00 & - \\
\hline Franja de Frontera & 569 & 2.284 .008 & 9.855 .729 & 6.950 .371 & 2.891 .667 & - & 196.036 & - & 209.050 & - & 555.324 & - \\
\hline
\end{tabular}

Los municipios del arco norte tienen por regla general baja accesibilidad geográfica, lo que implica dificultades añadidas para la atención a la salud (Figura 2). Estas dificultades están causadas por la gran extensión territorial, la dispersión poblacional y la falta de vías rápidas de acceso. El principal medio de comunicación es el fluvial, el único disponible en la mayoría de los municipios de la región. Los tiempos de los desplazamientos son grandes (contados en días y no en horas) con implicaciones tanto para la atención de salud, como para los procesos de propagación espacial de enfermedades, como se comprobó en la epidemia del cólera en la década de los noventa 13 .

El problema de la accesibilidad sólo se atenúa en dos sub-regiones del arco norte donde existe una red de autovías que conecta los municipios de la frontera con sus respectivas capitales provinciales y con los países colindantes. En Amapá, la BR-156 une Macapá a la Guayana Francesa, en Roraima, las BR-174 y 401 conectan Boa Vista a Manaus (Amazonas), a Venezuela y a la Guayana y en Acre, las BR-364 y 317 enlazan Río Branco con Porto Velho (Rondônia), Cobija (Pando, Bolivia) e Inãpari (Madre de Dios, Perú).
Las condiciones de vida del arco norte son las más desfavorables de toda la frontera (prácticamente todos los municipios excepto las capitales provinciales Boa Vista y Rio Branco se encuadran en esta categoría) (Figura 3). Este hecho se manifiesta en la situación de salud, pues fue la macrorregión de la frontera con peores indicadores de esperanza de vida y mortalidad infantil. El primero no supera los 65 años de vida en ninguna subregión, y el segundo alcanza sesenta óbitos por mil nacidos vivos en el Vale de Acre-Purus (SR-6) (Tabla 2).

El análisis de los datos de atención de salud apunta la escasez de infraestructura, recursos humanos y la baja cobertura de salud en esta región. Se confirma la estrecha relación existente entre condiciones de vida, situación de salud y el acceso a la atención.

La capacidad instalada de salud en el arco norte se concentra en las dos capitales provinciales (Boa Vista/Roraima y Rio Branco/Acre), lo que se pone de manifiesto en los mejores indicadores de atención de salud en las sub-regiones Campos de Río Branco (SR-2) y Valle de Acre-Purus (SR-6), donde están localizadas estas ciudades. La existencia 


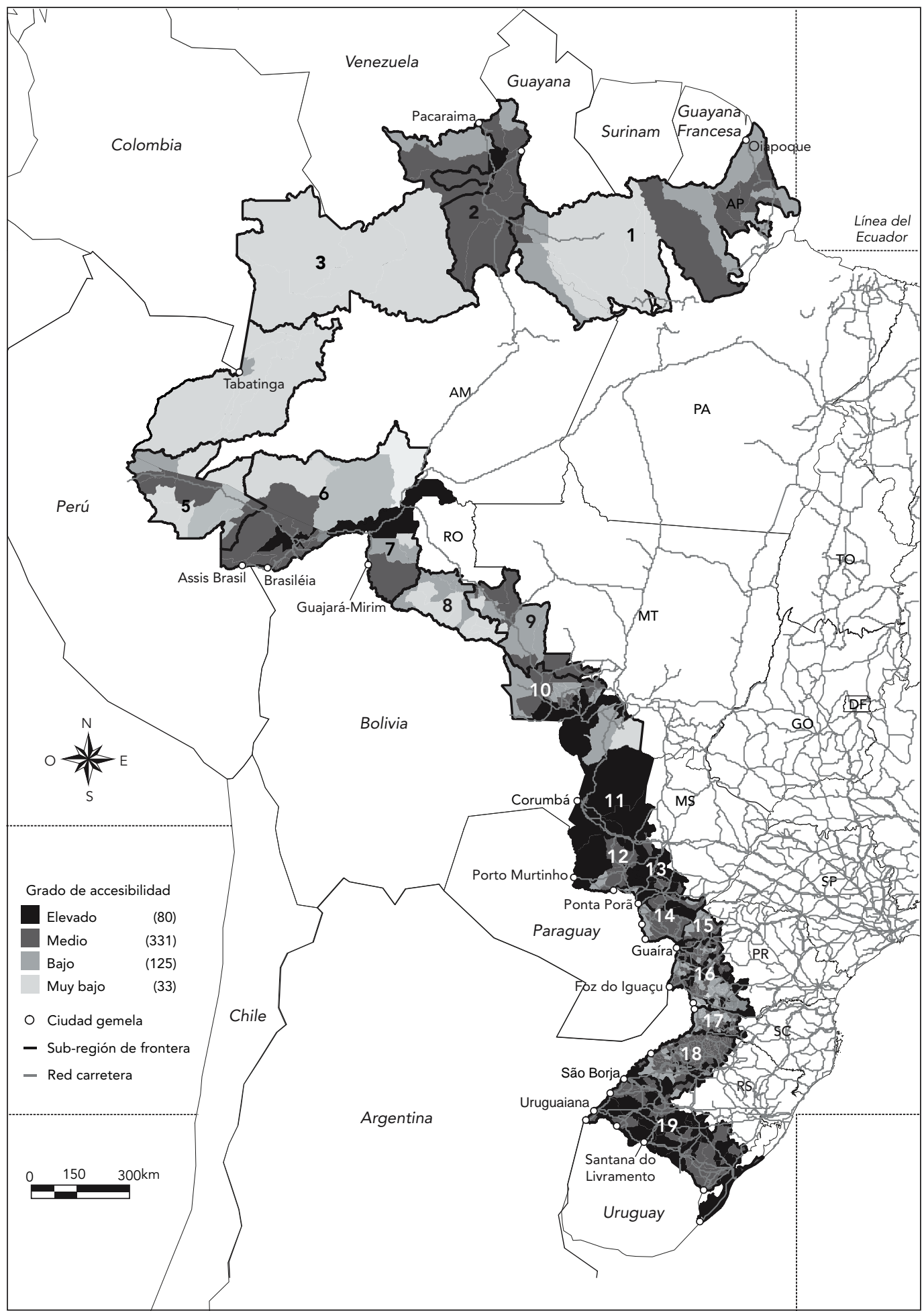

Fuente: Instituto de Investigaciones Económicas Aplicadas (http://www.ipeadata.gov.br, accedido el 10/Oct/2004); Guia 4 Rodas 11. Adaptado por Paulo C. Peiter. 
Figura 3

Franja de Frontera de Brasil: condiciones materiales de vida, 2000.

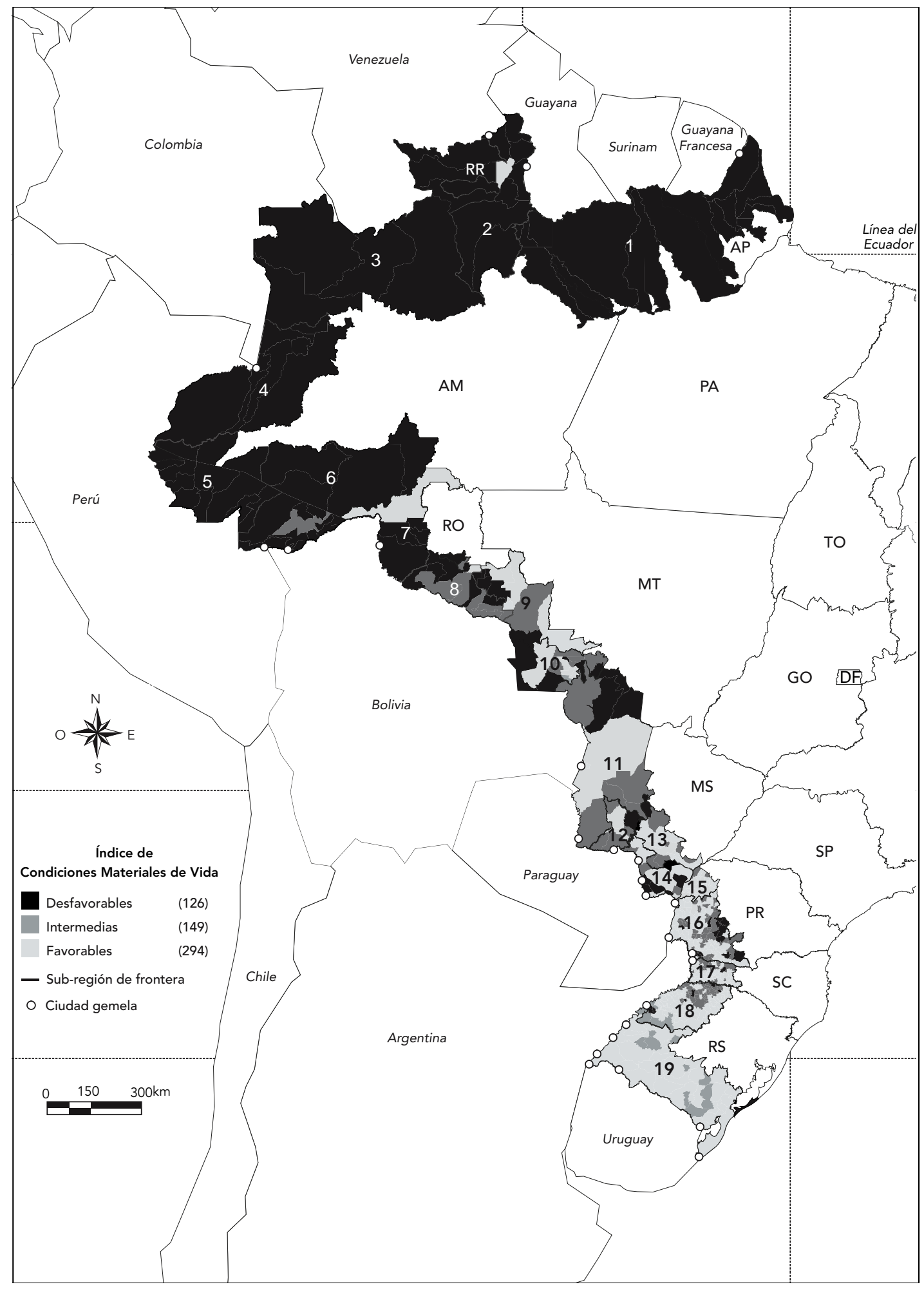

Fuente: Peiter 5 . 
Franja de Frontera (Brasil): aspectos de la asistencia a la salud, 1999-2000.

\begin{tabular}{|c|c|c|c|c|c|c|c|c|c|c|c|c|c|}
\hline \multirow[t]{2}{*}{ Sub-región } & \multicolumn{13}{|c|}{ Aspectos de la asistencia a la salud } \\
\hline & $\begin{array}{l}\text { Esperanza } \\
\text { de vida } \\
\text { al nacer }\end{array}$ & $\begin{array}{l}\text { Morta- } \\
\text { lidad } \\
\text { infantil }\end{array}$ & $\begin{array}{c}\text { Número } \\
\text { de hospi- } \\
\text { tales (2001) }\end{array}$ & $\begin{array}{c}\text { Camas/mil } \\
\text { habitantes } \\
\text { (1999) }\end{array}$ & $\begin{array}{l}\text { Ambula- } \\
\text { torios } \\
(2000)\end{array}$ & $\begin{array}{l}\text { Ambulato- } \\
\text { rios/10 mil } \\
\text { habitantes }\end{array}$ & $\begin{array}{c}\text { Número } \\
\text { de médicos } \\
\text { (1999) }\end{array}$ & $\begin{array}{l}\text { Médicos/ } \\
\text { mil } \\
\text { habitantes }\end{array}$ & $\begin{array}{l}\text { Enfer- } \\
\text { meros } \\
(1999)\end{array}$ & $\begin{array}{c}\text { Enfer- } \\
\text { meros/mil } \\
\text { habitantes }\end{array}$ & $\begin{array}{l}\text { Dentistas } \\
\text { (1999) }\end{array}$ & $\begin{array}{l}\text { Dentistas/ } \\
\text { mil } \\
\text { habitantes }\end{array}$ & $\begin{array}{c}\text { Cobertura } \\
\text { de } \\
\text { salud }\end{array}$ \\
\hline \multicolumn{14}{|l|}{ Arco Norte } \\
\hline \multicolumn{14}{|l|}{ Oiapoque- } \\
\hline $\begin{array}{l}\text { Tumucumaque } \\
\text { (SR-1) }\end{array}$ & e 66,19 & 39,20 & 17 & 2,19 & 158 & 5,29 & 115 & 0,39 & 63 & 0,21 & 32 & 0,11 & Baja \\
\hline \multicolumn{14}{|l|}{ Campos do } \\
\hline $\begin{array}{l}\text { Rio Branco } \\
\text { (SR-2) }\end{array}$ & 64,91 & 43,25 & 11 & 2,70 & 106 & 3,79 & 536 & 2,04 & 131 & 0,50 & 102 & 0,39 & Baja \\
\hline \multicolumn{14}{|l|}{ Parima-Alto } \\
\hline $\begin{array}{l}\text { Rio Negro } \\
\text { (SR-3) }\end{array}$ & 66,26 & 30,90 & 6 & 2,19 & 39 & 3,82 & 72 & 0,76 & 17 & 0,18 & 25 & 0,26 & Baja \\
\hline $\begin{array}{l}\text { Alto Solimões } \\
\text { (SR-4) }\end{array}$ & 64,17 & 27,09 & 6 & 1,39 & 16 & 0,93 & 72 & 0,44 & 16 & 0,10 & 36 & 0,22 & Baja \\
\hline $\begin{array}{l}\text { Alto Juruá } \\
\text { (SR-5) }\end{array}$ & 64,19 & 49,91 & 11 & 2,19 & 129 & 6,02 & 92 & 0,44 & 37 & 0,18 & 20 & 0,10 & Baja \\
\hline $\begin{array}{l}\text { Valle do } \\
\text { Acre-Purus } \\
\text { (SR-6) }\end{array}$ & 65 & 60,62 & 19 & 2,70 & 318 & 6,66 & 534 & 1,16 & 221 & 0,48 & 95 & 0,21 & Media \\
\hline \multicolumn{14}{|l|}{ Arco Central } \\
\hline \multicolumn{14}{|l|}{ (SR-7) } \\
\hline \multicolumn{14}{|l|}{ Frontera del } \\
\hline $\begin{array}{l}\text { Guaporé } \\
\text { (SR-8) }\end{array}$ & 66 & 38,57 & 17 & 2,30 & 238 & 10,29 & 141 & 0,63 & 26 & 0,12 & 18 & 0,80 & Alta \\
\hline \multicolumn{14}{|l|}{ Parecis (SR-9) } \\
\hline $\begin{array}{l}\text { Alto Paraguai } \\
\text { (SR-10) }\end{array}$ & 67 & 30,21 & 18 & 3,40 & 107 & 5,56 & 186 & 0,97 & 21 & 0,11 & 39 & 0,20 & Media \\
\hline $\begin{array}{l}\text { Pantanal } \\
\text { (SR-11) }\end{array}$ & 67 & 26,66 & 13 & 2,40 & 130 & 3,73 & 495 & 1,46 & 41 & 0,12 & 110 & 0,32 & Media \\
\hline $\begin{array}{l}\text { Bodoquena } \\
\text { (SR-12) }\end{array}$ & 70 & 18,25 & 6 & 1,90 & 27 & 2,72 & 96 & 0,98 & 6 & 0,06 & 35 & 0,36 & Alta \\
\hline $\begin{array}{l}\text { Dourados } \\
\text { (SR-13) }\end{array}$ & 68 & 30,5 & 25 & 3,20 & 118 & 3,34 & 582 & 1,67 & 45 & 0,13 & 103 & 0,30 & Media \\
\hline \multicolumn{14}{|l|}{ Cono Sur } \\
\hline $\begin{array}{l}\text { Matogrossense } \\
\text { (SR-14) }\end{array}$ & e 69 & 29,35 & 24 & 3,60 & 64 & 2,53 & 243 & 0,97 & 33 & 0,13 & 70 & 0,28 & Alta \\
\hline \multicolumn{14}{|l|}{ Arco Sur } \\
\hline $\begin{array}{l}\text { Portal do } \\
\text { Paraná (SR-15) }\end{array}$ & 69 & 28,22 & 5 & 4,30 & 222 & 7,35 & 450 & 1,50 & 152 & 0,51 & 141 & 0,47 & Alta \\
\hline Sudoeste do & 70 & 25,86 & 27 & 3,20 & 1.187 & 6,06 & 3.658 & 1,88 & 517 & 0,27 & 666 & 0,34 & Alta \\
\hline Paraná (SR-16) & & & & & & & & & & & & & \\
\hline Oeste de & & & & & & & & & & & & & \\
\hline $\begin{array}{l}\text { Santa Catarina } \\
\text { (SR-17) }\end{array}$ & 73 & 21,73 & 40 & 2,90 & 573 & 7,50 & 1.226 & 1,61 & 238 & 0,31 & 400 & 0,52 & Media \\
\hline
\end{tabular}

(continua) 
Tabla 2 (continuación)

\begin{tabular}{|c|c|c|c|c|c|c|c|c|c|c|c|c|c|}
\hline \multirow[t]{2}{*}{ Sub-región } & \multicolumn{12}{|c|}{ Aspectos de la asistencia a la salud } & \multirow[b]{2}{*}{$\begin{array}{c}\text { Cobertura } \\
\text { de } \\
\text { salud }\end{array}$} \\
\hline & $\begin{array}{l}\text { Esperanza } \\
\text { de vida } \\
\text { al nacer }\end{array}$ & $\begin{array}{l}\text { Morta- } \\
\text { lidad } \\
\text { infantil }\end{array}$ & $\begin{array}{c}\text { Número } \\
\text { de hospi- } \\
\text { tales (2001) }\end{array}$ & $\begin{array}{l}\text { Camas/mil } \\
\text { habitantes } \\
\text { (1999) }\end{array}$ & $\begin{array}{l}\text { Ambula- } \\
\text { torios } \\
(2000)\end{array}$ & $\begin{array}{l}\text { Ambulato- } \\
\text { rios/10 mil } \\
\text { habitantes }\end{array}$ & $\begin{array}{c}\text { Número } \\
\text { de médicos } \\
\text { (1999) }\end{array}$ & $\begin{array}{l}\text { Médicos/ } \\
\text { mil } \\
\text { habitantes }\end{array}$ & $\begin{array}{l}\text { Enfer- } \\
\text { meros } \\
\text { 1999) }\end{array}$ & $\begin{array}{c}\text { Enfer- } \\
\text { meros/mil } \\
\text { habitantes }\end{array}$ & $\begin{array}{c}\text { Dentistas } \\
\text { (1999) }\end{array}$ & $\begin{array}{l}\text { Dentistas/ } \\
\text { mil } \\
\text { habitantes }\end{array}$ & \\
\hline \multicolumn{14}{|l|}{ Noroeste do } \\
\hline $\begin{array}{l}\text { Rio Grande } \\
\text { do Sul (SR-18) }\end{array}$ & 3) & 19,45 & 45 & 4,20 & 964 & 6,96 & 2.983 & 2,15 & 582 & 0,42 & 475 & 0,34 & Alta \\
\hline Mitad Sur de & & & & & & & & & & & & & \\
\hline $\begin{array}{l}\text { Rio Grande } \\
\text { do Sul (SR-19) }\end{array}$ & 71 & 19,43 & 15 & 3,20 & 509 & 2,85 & 3.768 & 2,14 & 644 & 0,37 & 489 & 0,28 & Media \\
\hline
\end{tabular}

Fuente: Instituto Brasileño de Geografía y Estadística (IBGE) 9; Departamento de Informática del SUS (DATASUS. http://www.datasus.gov.br, accedido el 10/ Oct/2004).

de carreteras, uniendo las mencionadas capitales con los municipios de estas regiones, contribuye al mayor acceso a los servicios de sus poblaciones. En las demás sub-regiones del arco la escasez de recursos es grave. Muchos municipios no poseen médicos, y en la mayoría de las sub-regiones se dispone de menos de 1 médico por mil habitantes. Lo mismo ocurre con la disponibilidad de enfermeros y dentistas, insuficiente para atender a la población regional y muy por abajo del preconizado por la Organización Mundial de la Salud (Tabla 2). El índice de cobertura de salud también apunta la baja o muy baja cobertura en casi la totalidad de las sub-regiones, excepto en el Valle de Acre-Purus (SR-6).

Este cuadro de carencias y dificultades para la asistencia a la salud en el arco norte ocurre en una zona fronteriza, donde en diversos puntos, flujos de pacientes de los países vecinos sobrecargan los servicios locales. Estos flujos son más intensos en las seis ciudades gemelas del arco Oiapoque (Brasil)/Saint-Georges (Guayana Francesa); Bonfim (Brasil)/Lethem (Guayana); Pacaraima (Brasil)/Santa Elena de Uairén (Venezuela); Tabatinga (Brasil)/Leticia (Colombia); Benjamin Constant (Brasil)/Islandia (Perú); Assis Brasil (Brasil)/Iñapari (Perú)/Bolpebra (Bolivia); Brasiléia-Epitaciolândia (Brasil)/Cobija (Bolivia). En estas ciudades el tránsito transfronterizo de personas y mercancías forma parte del día a día y la búsqueda de servicios de salud involucra a habitantes de la zona de frontera nacionales y extranjeros. Según trabajos de campo realizados en estas ciudades en los años 2001 y 2004, la atención de salud básica, universal y gratuita en el lado brasileño de la frontera, es lo que más atrae a extranjeros de la frontera, sobre todo la atención prenatal y partos, los servicios de inmunización, y la obtención de medicamentos de distribución gratuita por el SUS. Los brasileños de esta región de la frontera buscan en los países vecinos la atención más especializada, consultas médicas y exámenes no disponibles en el lado brasileño. Sin embargo, esta situación es altamente variada dependiendo la ciudad y de las asimetrías existente entre ellas.

En el arco central de la frontera, el análisis de los indicadores seleccionados señaló una situación híbrida. En la parte septentrional del arco las condiciones se asemejan a las del arco norte, en la parte meridional la situación es más cercana a las encontradas en el arco sur.

El poblamiento de la parte septentrional del arco central presenta el mismo patrón disperso y menos denso del arco norte, en contrapartida, en la parte meridional se encuentran áreas con alta densidad poblacional y mayor accesibilidad geográfica. Viven en este arco 2.122.951 habitantes $(21,5 \%$ del total de la franja), casi un tercio en áreas rurales 9 . No obstante, incluso en estas áreas la accesibilidad es mejor que en el arco norte, pues existe una red viaria relativamente desarrollada en toda la región (Tabla 1).

En las sub-regiones Madera-Mamoré (SR-7), Frontera del Guaporé (SR-8), Chapada de los Parecis (SR-9), y Alto Paraguai (SR-10), en los Estados de Rondônia y Mato Grosso, la circulación viaria se estructura alrededor del eje de la BR364 (Cuiabá-Porto Velho). En las sub-regiones de Pantanal (SR-11), Bodoquena (SR-12), Dourados (SR-13) y Cono-Sur Matogrossense (SR-14), en el Estado de Mato Grosso do Sul hay una densa red de autovías federales y provinciales bien articuladas, que proporcionan mayor accesibilidad geográfica a sus municipios (Figura 2).

En las últimas décadas, fue intenso el crecimiento poblacional de este arco de la frontera, que estuvo vinculado al proyecto de colonización rural y ampliación de la frontera agrícola brasileña (expansión de la ganadería y del cultivo de 
soja). Por ello, el arco central recibió un gran número de emigrantes sureños y procedentes del Nordeste. En los dos últimos años de la década de los noventa llegaron a la región cerca de 75 mil emigrantes, que se dirigieron sobre todo hacia las sub-regiones: Madera-Mamoré (SR-7 - 19.784 emigrantes); Frontera del Guaporé (SR-8 - 10.894 emigrantes) y Chapada de los Parecis (SR-9 - 12.286 emigrantes) (Tabla 1). Esto representó la llegada de una población potencialmente más vulnerable y un profundo proceso de transformación económica, social y ambiental. Este proceso tuvo consecuencias desastrosas para la salud de los grupos más vulnerables, como los indígenas y para los propios emigrantes recién llegados (asentados), como por ejemplo la explosión de malaria en la región en lo largo de los años ochenta 14 .

Innumerables ciudades surgieron a partir de este proceso, y otras crecieron aceleradamente, conformando una red urbana más "desarrollada" que la del arco norte (en términos de número de ciudades y de tamaño), con varios núcleos urbanos con más de 50 mil habitantes. Se destaca en este segmento de la frontera la capital de Rondônia, Porto-Velho con más de 200 mil habitantes, donde se concentra la infraestructura de salud regional; y la influencia de Cuiabá (fuera de la franja), Cáceres y Corumbá como ciudades articuladoras de los principales flujos transfronterizos en la porción septentrional del arco central. Sin embargo, es la zona de frontera BrasilParaguay, en la parte meridional de este arco, la que presenta mayor circulación transfronteriza, es decir, donde la frontera es más "porosa". En este trecho son importantes los problemas relacionados con el tráfico de drogas, contrabando y violencia 15. Estos problemas tienen impactos significativos en la atención de salud en la región.

Las condiciones de vida, situación de la salud y atención de salud en el arco central de la frontera pueden ser caracterizadas mejor como "transicionales" entre el patrón de la frontera amazónica y el de la frontera sureña.

Los indicadores de condiciones de vida presentan variaciones acentuadas entre sub-regiones e incluso en el interior de éstas. Predominan las condiciones de vida desfavorables en las subregiones situadas en la parte septentrional del arco central (con excepción del Municipio de Porto Velho en la sub-región Madera-Mamoré), mientras que en la porción meridional las condiciones de vida son mejores (Figura 3). La situación de salud sigue, en líneas generales, este mismo patrón. La esperanza de vida al nacer varía de 65 años en la sub-región Madera-Mamoré (SR-7 - extremo norte del arco) a 70 años en la sub-región Bodoquena (SR-12 - al sur del arco central). La mortalidad infantil varía de 38,57 óbitos por mil nacidos vivos en la sub-región frontera del Guaporé (SR-8), a 18,25 óbitos por mil nacidos vivos en la sub-región Bodoquena (SR-12 -el valor menor de la franja de frontera) (Tabla 2).

Los recursos humanos de salud y la atención de complejidad media están concentradas en tres polos: en la parte norte, en Porto Velho (SR-7 - Madeira-Mamoré), en la parte central, en Corumbá (SR-11 - Pantanal) y en la parte sur en Dourados (SR-13 - Dourados). La red hospitalaria tiene una distribución más uniforme, siendo que la sub-región de Alto Paraguai es la que presenta mayor oferta de camas (3,4/mil habitantes) (Tabla 2). La distribución geográfica de la red ambulatoria es desequilibrada. En la sub-región de la Frontera del Guaporé (SR-8) la relación es de 10,3 ambulatorios por 10 mil habitantes, mientras que en la sub-región del Cono Sur Matogrossense (SR-14) es de 2,53/10 mil habitantes.

El índice de cobertura de salud muestra una situación más favorable en todo el arco, con una variación de la cobertura entre media y alta.

Los mejores indicadores del arco central retratan una situación más favorable para la asistencia a la salud en la región, con pequñas diferencias entre las sub-regiones. A pesar de ello, las mayores conexiones de la región e intensidad de las interacciones transfronterizas en este arco, sobre todo en su mitad sur, convierte a menudo esta región en objetivo de las redes criminales del tráfico de drogas y contrabando (inclusive de armas), lo que aumenta la vulnerabilidad de sus poblaciones y supone serios problemas para la atención de salud.

El arco sur es la macrorregión más populosa de la frontera con 6.186 .840 habitantes $(63 \%$ del total) y también donde la densidad rural es más elevada (Tabla 1). Pese a esto, a diferencia de los demás arcos, el segmento sur presenta un cuadro poblacional más estable. La composición de la población por franjas de edad y sexo es distinta, pues hay una mayor proporción de personas con más de 60 años de edad y ligera predominancia de mujeres. La red urbana es la más desarrollada con un razonable número de ciudades medianas (más de 100 mil habitantes) como Foz do Iguaçu, Cascavel, Bagé, Pelotas y Rio Grande, distribuidas uniformemente a lo largo de la frontera, además de poseer el mayor número de ciudades gemelas de la frontera (12 en total). La profusión de ciudades gemelas retrata, no sólo mayor densidad poblacional, sino también mayor intensidad de las interacciones transfronterizas de las zonas de frontera entre Brasil, Paraguay, Argentina y Uruguay. Las inte- 
racciones transfronterizas en este trecho de la frontera tienen una larga historia, pero han pasado por profundas transformaciones desde la creación del MERCOSUR en 1991.

La mayor fragmentación del arco sur que posee 403 municipios se debe entre otros motivos al proceso histórico de ocupación de la región, basado en pequeñas propiedades rurales, que a su vez propició una mejor distribución de renta en la región con reflejos directos en las condiciones de vida de su población. Resulta que de los 403 municipios del arco solamente 10 (2,5\%) se sitúan en el apartado de condiciones de vida bajas (Figura 3).

La situación de salud refleja las mejores condiciones de vida en el arco. La esperanza de vida supera los 70 años de edad en tres de las cinco sub-regiones. La mortalidad infantil es inferior a 30 óbitos por mil nacidos vivos en todas ellas, alcanzando el nivel más bajo (19,43 óbitos por mil nacidos vivos) en la mitad sur de Rio Grande do Sul (SR-19) (Tabla 2).

Estos resultados reflejan, también, una mayor disponibilidad de recursos para la atención de salud en el arco. El análisis de los indicadores apuntó mayor disponibilidad de médicos, con un porcentaje de médicos por mil habitantes superior al promedio nacional en casi todas las subregiones, excepto en el Portal de Paraná (SR-15) (1,5 médicos/mil habitantes). Lo mismo ocurrió con la disponibilidad de enfermeros y dentistas (Tabla 2). El índice de cobertura de salud también señaló una mejor situación de la atención de salud en el arco sur, con alta cobertura en la mayor parte de las sub-regiones. A todos estos aspectos favorables se suma la mayor accesibilidad geográfica de los municipios del arco, que presenta la red viaria más densa de la frontera y conectada por autovías federales con las principales capitales del Sur y Sudeste de Brasil. Asimismo, las distancias entre núcleos municipales y entre éstos y sus respectivas capitales provinciales son relativamente menores que en los demás arcos de la frontera.

Por otro lado, la mayor intensidad de las interacciones transfronterizas a lo largo del arco sur dificulta el control de la salud y la demanda de los extranjeros en las fronteras sobrecarga los servicios de salud de los municipios de la región (en particular en las ciudades gemelas).

El arco norte fue sin duda el que presentó la situación más crítica para la atención de salud en toda la franja fronteriza, con relevancia en las sub-regiones Oiapoque-Tumucumaque (SR-1), Parima Alto-Río Negro (SR-3), Alto Solimões (SR-4) y Alto Juruá (SR-5). En estas sub-regiones la combinación de condiciones de vida desfavorables con la escasez de recursos huma- nos, infraestructura de salud y baja accesibilidad geográfica de los municipios se manifiesta en los elevados índices de mortalidad infantil y en la baja esperanza de vida al nacer de la población. También son elementos agravantes: el proceso de ocupación desordenada de la región con la llegada de inmigrantes (agricultores, madereros, buscadores de metales y piedras preciosas, etc.) de varias partes del país; los conflictos derivados de la disputa por tierra con las poblaciones autóctonas (en particular con los indígenas); y la elevada movilidad interna y transfronteriza, que dificulta el control y la implementación de acciones de salud.

Entre el conjunto de indicadores utilizados para la descripción de la situación de la atención de salud en la región, la falta de médicos y de enfermeros en varios municipios es el que más llama la atención. Esta falta de profesionales cualificados de salud se agrava en las ciudades gemelas y en áreas de mayor intensidad de interacciones transfronterizas, donde los servicios de salud ya saturados y funcionando en condiciones precarias reciben contingentes adicionales de pacientes originarios de diversas localidades fronterizas de los países vecinos. Sin duda estos aspectos aumentan la vulnerabilidad en la región.

El arco central es el más heterogéneo de la frontera, con situaciones favorables para la atención de salud en las sub-regiones de la Chapada de los Parecis (SR-9), Alto Paraguai (SR-10), Pantanal (SR-11), Dourados (SR-13) y Cono Sur Matogrossense (SR-14) y críticas en las sub-regiones Madera-Mamoré (SR-7), Frontera del Guaporé (SR-8).

En el cómputo general el arco sur fue el que presentó mejores indicadores de atención a la salud. Los demás indicadores también son ampliamente favorables en este arco de la frontera, y mucho, aunque sea el segmento de la frontera con mayor intensidad de interacciones fronterizas, su mejor infraestructura y mayor disponibilidad de personal cualificado son elementos que capacitan la región para enfrentar estos desafíos.

\section{Discusión}

El estudio mostró una frontera altamente heterogénea. El arco norte es el de situación más crítica, teniendo como principal desafío extender la atención a la totalidad de sus poblaciones, mejorando la distribución espacial de los servicios (cobertura y accesibilidad geográfica), y aumentando la capacidad instalada, inclusive para la atención de media y alta complejidad. Es un reto inaplazable la búsqueda y contratación de médicos, enfermeros y dentistas en la región. 
En el arco central los desafíos son superar los problemas derivados del proceso de ocupación desordenada, de la rápida transformación del territorio regional (expansión de la frontera agrícola), y los problemas relacionados con el tráfico de drogas, contrabando y otras actividades ilegales que aumentan la violencia y la vulnerabilidad de sus poblaciones. La atención a la salud tiene que estar preparada para estar a la altura de estos problemas.

En el arco sur el mayor desafío es sobrellevar la demanda creciente de ciudadanos fronterizos de los países del MERCOSUR y mantener la calidad de la atención.

Además, el perfil poblacional de este arco, con población más anciana y predominantemente femenina, plantea distintos desafíos para la atención de la salud en la región.

Este aspecto puede todavía contar con un elevado número medio de consultas, utilizado en el índice compuesto de cobertura de salud, perjudicando la comparación con los demás arcos, por lo tanto, existen precauciones que deben ser tomadas cuando se utilizan indicadores compuestos que pueden sesgar algunos resultados.

\section{Resumen}

Este estudio se propone identificar espacios críticos en la atención a la salud de la Franja Fronteriza de Brasil. Para ello se realizó una caracterización de la distribución de los recursos de salud por municipio y regiones fronterizas en 1999-2000, posteriormente relacionada con las condiciones de vida, situación de salud, accesibilidad geográfica y otros aspectos de la dinámica fronteriza. Se utilizaron datos secundarios de grandes bancos de datos nacionales de salud y geografía, completados con datos primarios recogidos sobre el terreno. Para el análisis de los datos y producción de mapas se utilizaron Sistemas de Información Geográfica. La Franja de Frontera se mostró heterogénea en los aspectos considerados. El segmento que comprende
Otra limitación del estudio se produce por la utilización de datos secundarios, cuya recogida es precaria en muchos de los municipios estudiados, sobre todo en el arco norte y central. Parte de estas limitaciones se resolvió con los trabajos de campo, donde fue posible verificar y completar la información.

A pesar de estas limitaciones este tipo de análisis panorámico puede contribuir a la creación de un cuadro aproximado de los problemas y necesidades de salud de las poblaciones fronterizas y la disponibilidad de recursos de salud en las diferentes regiones de la frontera. Pese a que no se puedan sacar conclusiones definitivas de estos estudios, son guías útiles para nuevas investigaciones más profundas en regiones específicas, donde las evidencias preliminares apuntan la existencia de situaciones más críticas en la atención a la salud.

El estudio apuntó la necesidad de políticas de salud en la frontera que corrijan las distorsiones de la distribución de los recursos de salud observadas, considerando las desigualdades regionales de condiciones de vida, situación de salud y accesibilidad, así como las particularidades de las regiones fronterizas. los municipios situados en la frontera norte presentó las condiciones más desfavorables constituyéndose en la región más crítica para la atención a la salud. El segmento central presentó una situación intermedia, con una proporción equilibrada de sub-regiones en situación crítica y no-crítica. El segmento sur presentó una situación satisfactoria en todas las sub-regiones. La heterogeneidad de la salud en la Franja Fronteriza mostró la necesidad de un enfoque específico que considere sus diferentes regiones y las particularidades de la dinámica fronteriza.

Áreas Fronterizas; Servicios de Salud; Condiciones Sociales; Atención a la Salud 


\section{Referencias}

1. Gallo E, Costa L. SIS-MERCOSUL: uma agenda para a integração. Brasília: Organização Pan-Americana da Saúde; 2004. (Série Técnica: Projeto de Desenvolvimento de Sistemas e Serviços de Saúde, 9).

2. Rumley D, Minghi J. The geography of border landscapes. London: Routledge; 1991.

3. Prescott JRV. Political frontiers and boundaries. London: Allen \& Unwin; 1987.

4. House JW. The frontier zone: a conceptual problem for policy makers. International Politics Science Review 1980; 1:456-77.

5. Peiter PC. A geografia da saúde na faixa de fronteira continental do Brasil na passagem do milênio [Tesis de Doctorado]. Rio de Janeiro: Programa de Pós-Graduação em Geografia, Universidade Federal do Rio de Janeiro; 2005.

6. Kammel WW. Dilemas sanitarios en las fronteras: un desafío mundial. Foro Mundial de la Salud 1997, 18:11-20.

7. Machado LO. Estado, territorialidade, redes. Cidades-gêmeas na zona de fronteira sul-americana. In: Silveira ML, organizador. Continente em chamas. Globalização e território na América Latina, Rio de Janeiro: Editora Civilização Brasileira; 2005. p. 243-84.

8. Ministério da Integração Nacional. Proposta de reestruturação do programa de desenvolvimento da faixa de fronteira. Brasília: Ministério da Integração Nacional; 2005.
9. Instituto Brasileiro de Geografia e Estatística. Censo demográfico 2000: características da população e dos domicílios. Resultados do universo. Rio de Janeiro: Instituto Brasileiro de Geografia e Estatística; 2002.

10. Instituto Brasileiro de Geografia e Estatística. Pesquisa de assistência médico-sanitária (AMS) Rio de Janeiro, 1999 e 2002. Rio de Janeiro: Instituto Brasileiro de Geografia e Estatística; 2002.

11. Guia 4 Rodas. São Paulo: Editora Abril; 2000.

12. Leonardi VPB. Fronteiras Amazônicas do Brasil: saúde e história social. São Paulo: Editora Marco Zero; 2000.

13. Toledo LM. O espaço do cólera: Determinantes sociais e regulação ambiental dos caminhos de uma epidemia [Tesis de Doctorado]. Rio de Janeiro: Escola Nacional de Saúde Pública, Fundação Oswaldo Cruz; 1996.

14. Bretas G. A determinação da malária no processo de ocupação da fronteira agrícola [Dissertación de Maestría]. Rio de Janeiro: Escola Nacional de Saúde Pública, Fundação Oswaldo Cruz; 1990.

15. Machado LO. Comércio ilícito de drogas e a geografia da integração financeira. Uma simbiose? In: Castro I, organizador. Brasil. Questões atuais da reorganização do território. Rio de Janeiro: Editora Bertrand Brasil; 1996. p.185-220.

Recibido el 19/Jun/2006

Versión final presentada el 25/Abr/2007

Aprobado el 03/May/2007 